\title{
ANÁLISE DO COMPORTAMENTO DA FO EM ENUNCIADOS NUMÉRICOS DE CPF NO PORTUGUÊS BRASILEIRO
}

\section{ANALYSIS OF THE FO BEHAVIOR IN CPF'S NUMERICAL UTTERANCES IN BRAZILIAN PORTUGUESE}

\begin{abstract}
Ayane Nazarela Santos de Almeida ${ }^{15}$ Miguel Oliveira $\mathrm{Jr}^{16}$

RESUMO: O presente artigo objetiva analisar o comportamento da frequência fundamental (f0) na elocução de agrupamentos numéricos de CPF (código de pessoa física) no português do Brasil. Investiga a forma mais recorrente para números de CPF a partir de características prosódicas específicas que se revelam na segmentação dos agrupamentos e caracteriza as unidades prosódicas constituindo evidências de que os números nominais apresentam uma estrutura bem definida. Todos os dados utilizados nesse experimento são reais no intuito de prezar pela apresentação original objetivando testar a relação entre a disposição gráfica e a forma enunciada. Motivados pela literatura (SWERTS, 1997; OLIVEIRA JR, 2000; OUDEN, 2004), selecionamos para a análise as variáveis prosódicas de variação da f0, declínio da f0 e diferença de tom. Verificamos que foi recorrente o tom de fronteira alto marcando as fronteiras prosódicas e baixo no final do enunciado; que houve uma declinação da f0 no interior das unidades prosódicas e esta foi ainda mais significativa ao considerarmos o enunciado como um todo. Tais resultados nos levaram a concluir que os números de CPF, produzidos de forma espontânea, são integrados a uma estrutura subjacente e que ao fazerem uso sistemático de vários elementos prosódicos, os falantes tratam os agrupamentos numéricos da mesma forma que enunciados declarativos típicos em termos de sua estrutura prosódica.
\end{abstract}

PALAVRAS-CHAVE: Prosódia. Frequência Fundamental. Números Nominais. Português Brasileiro

\begin{abstract}
The present paper aims to analyze the behavior of fundamental frequency (f0) in the utterance of CPF's numerical groupings (individual taxpayer registry identification) in Brazilian Portuguese. It investigates the most recurrent form for CPF's numbers from specific prosodic characteristics that are revealed in the segmentation of groupings and it characterizes the prosodic units constituting evidence that the nominal numbers present a well defined structure. All the data used in this experiment are real so that the original presentation could be appreciated in order to test the relationship between graphic layout and the spoken form. Motivated by the literature (SWERTS, 1997; OLIVEIRA JR, 2000; OUDEN, 2004), we selected for the analysis the prosodic variables of variation of $\mathrm{f0}$, declination of $\mathrm{f0}$, and difference of tone. We found a recurring high boundary tone, marking the prosodic boundaries and with a low tone at the end of the utterance; that there was a declination of f0 within the prosodic units and this was even more significant when considering the utterance as a whole. These results led us to conclude that the CPF's numbers, produced in a spontaneous way, are integrated into an underlying structure and that in making systematic use of several prosodic elements, the speakers treat the numerical groupings in the same way as declarative sentences in terms of their prosodic structure.
\end{abstract}

KEYWORDS: Prosody. Fundamental Frequency. Nominal Numbers. Brazilian Portuguese

\section{Introdução}

Em português, como em qualquer outra língua, existem estruturas linguísticas regulares ou fixas que constituem expressões cujo significado depende da observação de características segmentais e suprassegmentais de seus constituintes. Os agrupamentos numéricos, utilizados para nomear entidades, distinguindo-as entre si (WIESE, 2003), são exemplos dessas estruturas regulares. O presente trabalho apresenta uma descrição da estrutura e das características entoacionais de um agrupamento numérico muito produtivo no português brasileiro: aquele associado ao cadastro de pessoa física (CPF).

\footnotetext{
15 Doutora em Linguística. Professora do curso de Licenciatura em Letras: Língua Portuguesa/Libras/Língua Inglesa da Universidade Federal do Recôncavo da Bahia. E-mail: ayanesantos@hotmail.com

${ }^{16}$ Doutor em Linguística. Professor da Faculdade de Letras (FALE-UFAL) e do Programa de Pós-graduação em Letras e Linguística da Universidade Federal de Alagoas. E-mail: miguel@fale.ufal.br
} 
Com o avanço da tecnologia, tem-se tornado cada vez mais frequente o uso de sistemas automatizados de síntese e reconhecimento de fala nos mais diversos âmbitos de aplicação. Assim, por exemplo, já há uma quantidade significativa de serviços que faz uso de informações derivadas de conjuntos numéricos conectados para os mais variados fins: ativação de cartões de crédito, informações bancárias, consultas a listas telefônicas e marcações de reservas são alguns dos exemplos mais comuns de tal aplicação. Em muitos casos, entretanto, a performance desses sistemas tem sido considerada sofrível, ora por não processarem corretamente a fala espontânea (no caso de sistemas de reconhecimento de fala), ora por não oferecerem uma produção próxima à fala natural (no caso dos sistemas de síntese de fala).

É ainda muito comum, por exemplo, que usuários de linhas telefônicas se queixem dos sistemas automatizados com que em geral têm de interagir ao telefone, uma vez que simplesmente não reconhecem aquilo que falam. Nessas situações, escutam, em geral, um indefectível e frustrante feedback, do tipo: "não entendi". As queixas também se referem ao modo como certos agrupamentos numéricos são enunciados por sistemas de síntese de fala. A maneira como esses sistemas agrupam números e a prosódia que utilizam para enunciá-los nesses casos em nada se assemelham à sua enunciação natural. Isso tem um impacto significativo em termos de compreensão desses constituintes, o que é evidentemente algo indesejável no que concerne ao que se espera de sistemas automatizados de síntese de fala.

Esses problemas devem-se em parte ao fato de esses sistemas geralmente serem baseados em dados controlados e/ou resultantes de análises impressionistas e não em dados resultantes de análises de dados de fala natural e espontânea. Deriva daí a necessidade premente de informações que visem ao aperfeiçoamento dos sistemas computacionais automáticos ora em uso.

Avanços nessa área têm sido obtidos em decorrência da descrição da estrutura prosódica de números naturais nas mais diversas línguas, tais como o inglês (RAHIM et al., 2001), o alemão (BAUMANN; TROUVAIN, 2001; CREER, 2002), o espanhol (LÓPEZ et al., 1998), o francês (MOKBEL et al., 1997), o japonês (AMINO; OSANAI, 2011), o húngaro (OLASZI, 2000), o chinês (WANG; SENEFF, 1998) e o português europeu (RODRIGUES, 2001). Até o momento, poucos são os trabalhos sobre o português do Brasil que descrevem, de forma sistemática e abrangente, as várias características acústicas da organização de números naturais em estruturas pré-estabelecidas (ALMEIDA, 2017; OLIVEIRA JR; SILVA, 2017; MUSILIYU, 2014).

O presente estudo visa a contribuir nesse sentido, apresentando uma descrição do comportamento da f0 de agrupamentos numéricos em uma estrutura fixa (o cadastro de pessoa física brasileiro), tal como enunciada espontaneamente. Objetiva-se também, e a partir dos resultados dessa análise, propor um modelo de descrição e geração a ser usado para aprimorar um sistema de síntese e reconhecimento de fala para o português do Brasil. O estudo sobre a prosódia de números nominais de CPF integra, dentre outras, questões de estruturação, agrupamento, entoação e constitui uma contribuição para a pesquisa sobre os números falados em geral, que claramente desempenham um importante papel na nossa interação cotidiana. ${ }^{17}$

Para Reed (2011), o primeiro passo em qualquer análise prosódica é a identificação das unidades prosódicas, unidades linguísticas definidas por aspectos suprassegmentais da fala. São fragmentos de fala que constituem uma unidade caracterizada pela presença de fronteiras que os delimitam. Apesar de caracterizadas com base em uma variedade de critérios, a intuição tem papel relevante na identificação das fronteiras que limitam essas unidades. De acordo com Chafe (1994), são as características acústicas primitivas, identificadas intuitivamente, que definem as fronteiras prosódicas que delimitam essas

\footnotetext{
${ }^{17} \mathrm{O}$ trabalho é fruto do projeto "A prosódia de agrupamentos numéricos no português do Brasil, financiado pelo CNPq" (Processo: 405661/2012-6).
} 
unidades da fala. Além da pausa, outros parâmetros prosódicos, como aqueles analisados na presente investigação, relacionados à variação da frequência fundamental (f0), fornecem pistas para a demarcação/identificação dessas fronteiras.

\section{Unidade prosódica}

Ao analisarmos, no nível da sentença, se determinadas palavras pertencem ou não a um mesmo constituinte linguístico, podemos considerar as relações sintáticas, semânticas e/ou pragmáticas que existem entre elas, garantindo um alto nível de coesão e coerência. De forma semelhante, as relações que se estabelecem entre enunciados definem se são pertencentes a um discurso, uma estrutura linguística maior. Dessa maneira, são unidades menores, dispostas hierarquicamente e que mantêm orientação semelhante, as responsáveis por compor a estrutura linguística do discurso (OLIVEIRA JR; CRUZ; SILVA, 2012).

No nível da prosódia, são parâmetros como pausa, tons de fronteira, diferença de pitch, por exemplo, que irão sinalizar auxiliando a delimitação das macroestruturas discursivas em textos orais. Ressalta-se, porém, que da mesma forma que falantes de qualquer língua podem usar vários meios acústicos para atribuir estrutura ao texto falado, podendo, por exemplo, dividi-lo em partes menores usando pausas ou movimentos de entoação, e/ou marcando palavras altamente importantes como mais proeminentes (DONZEL, 1999), os ouvintes também são capazes de detectar essas estruturas: eles geralmente têm noções sobre a estrutura do texto a que estão sendo expostos. Ao ouvir alguém contar uma história, por exemplo, percebe-se quando o orador terminou uma parte de sua história (SWERTS; GELUYKENS, 1994; CHAFE, 1994; OLIVEIRA JR, 2000).

Isso indica que os ouvintes também são capazes de dizer quando novas unidades da fala começam, e quando terminam, e também são capazes de dizer qual informação é menos importante, ainda que não sejam treinados para esse fim. Isso foi demonstrado, por exemplo, nas pesquisas de Swerts e Geluykens (1994), Blaauw (1995), Swerts (1997), Oliveira Jr., Cruz e Silva (2012) e Barnwell (2013). Ou seja, ao utilizar-se da prosódia para estruturar o fluxo da informação discursiva, demarcando-a, o falante valida a relevância das variáveis prosódicas sob a perspectiva da percepção do ouvinte. Alguns estudos prévios já investigaram a importância das variáveis prosódicas na percepção do discurso falado (COLLIER, 1993; SWERTS; COLLIER; TERKEN, 1994; SWERTS; GELUYKENS, 1994; SWERTS, 1997).

A língua falada é segmentada em unidades menores que Chafe (1994) conceitua como expressões linguísticas de informação, inicialmente ativadas na consciência do falante e em seguida na do ouvinte através do enunciado e apesar de identificáveis com base em uma variedade de critérios, a intuição tem papel relevante na identificação das fronteiras que limitam esses elementos.

Não há consenso, entretanto, em torno da definição dessas unidades menores da fala e concepções distintas admitem diferentes formas de nomear tais fragmentos (BARTHWEINGARTEN, 2013). Apesar de tantas nomenclaturas, Reed (2010) afirma que todos os termos se referem à noção básica de uma unidade linguística definida por aspectos suprassegmentais da fala. Ou seja, são trechos de fala que constituem um enunciado e marcam a presença de fronteiras que delimitam essas unidades menores.

Embora o termo unidade entoacional seja comumente conhecido na literatura (LIEBERMAN, 1967; BOLINGER, 1989; OLIVEIRA JR, 2000; BARTH-WEINGARTEN, 2013), adotamos nesse trabalho o termo unidade prosódica pois entendemos que, ao fragmentar a fala em unidades menores, ainda que intuitivamente, utilizamos outros parâmetros prosódicos além da entoação para demarcar as fronteiras que as delimitam. Além disso, evitamos qualquer possível confusão entre os termos entoação e prosódia que não consideramos sinônimos. 
O discurso espontâneo, mesmo que seja menos estruturado e caracteristicamente contenha mais disfluências e hesitações, tem em linhas gerais uma estrutura assim como um material mais cuidadosamente estruturado. A mesma construção global que se aplica em sintagmas, orações, sentenças e parágrafos. No discurso espontâneo, os ouvintes também mantêm o status da informação dos conceitos introduzidos anteriormente pelo falante (DONZEL, 1999).

Nesse sentido, Cruttenden (1997[1986]) destaca que ao considerarmos a fala espontânea, qualquer segmentação não é tão evidente assim, devido a sua natureza fragmentada, com hesitações, repetições, falsos começos e frases incompletas. Desse modo, Oliveira Jr (2000) esclarece que por influenciar a percepção da estrutura do discurso, é natural que a prosódia seja usada como guia pelo investigador, na identificação de tal estrutura, no entanto, ao optar por esse tipo de análise corre-se o risco de incorrer em circularidade (SWERTS; COLLIER, 1992; SWERTS; GELUYKENS, 1994; SWERTS; COLLIER; TERKEN, 1994; SWERTS, 1997).

Ao explicar circularidade, Ladd (1986) afirma que se algo é estruturalmente uma unidade prosódica, então terá necessariamente fronteiras prosódicas, e se algo tem fronteiras prosódicas, então será uma unidade prosódica. Dessa forma, se o objetivo dessa pesquisa é identificar o comportamento da f0 na estruturação da informação numérica, procuramos minimizar o efeito da circularidade subjacente a tal análise, partindo de uma concepção perceptual para a delimitação das unidades e submetendo a examinadores para posterior análise dos correlatos acústicos, seguindo orientações de Oliveira Jr (2012). Tal procedimento, bem como outros métodos utilizados nessa pesquisa estão descritos a seguir.

\section{Procedimentos metodológicos}

Os dados utilizados no presente estudo foram resultantes de uma coleta realizada em áudio e obtidos através de uma pequena entrevista durante a qual os participantes foram solicitados a falar livremente informações pessoais, tais como: sua cor favorita, sua preferência no esporte, onde reside, seus telefones (fixo e móvel), número de CPF e o código do CEP de sua residência. Para essa tarefa, os participantes não consultaram seus dados pessoais, eles enunciaram os números da forma como haviam memorizado. Por essa razão, apesar de haver controvérsias acerca do que caracteriza uma enunciação espontânea, consideramos que os dados pessoais foram enunciados pelos participantes de forma natural, espontânea.

A coleta de dados seguiu critérios técnicos e recomendações de Oliveira Jr (2014) para sua adequada realização. Os registros das gravações foram feitos em formato PCM, não-comprimido (gravados em formato wav), com taxa de amostragem de $96 \mathrm{kHz}$ e 32 bits por amostra, utilizando-se um microfone tipo headset DPA Headband 4066 e um gravador digital de flash Marantz PMD661. A adoção de tais medidas garantiu arquivos de áudio de alta qualidade e, consequentemente, condições para análises acústicas acuradas. ${ }^{18}$ Além disso, a utilização do microfone headset propiciou a minimização do risco de distâncias diferentes entre a boca do participante e o microfone em cada gravação, o que afetaria nossas medidas acústicas (OLIVEIRA JR, 2000).

Os arquivos de áudio, gerados a partir das gravações das entrevistas para coleta de dados pessoais, foram segmentados individualmente no aplicativo computacional Praat (BOERSMA, 2001). Esse procedimento gerou arquivos de áudio para cada agrupamento

\footnotetext{
${ }^{18}$ Apenas uma gravação (Informante F65) foi excluída da análise por não ter apresentado qualidade acústica adequada.
} 
numérico enunciado por cada participante da pesquisa, inclusive CPF, objeto de análise no presente estudo.

Após gerar os arquivos de áudio de todos os dados, realizamos a segmentação e a anotação dos mesmos, também utilizando o Praat, de modo a identificar as unidades prosódicas, seguindo orientações de Reed (2011). Então, a anotação foi feita considerando a identificação dessas unidades e para tanto, adotamos uma abordagem intuitiva, utilizando a percepção, enquanto falante nativo da língua, para segmentar os agrupamentos numéricos em unidades menores, seguindo autores que utilizaram a mesma abordagem em sentenças não numéricas, tais como Collier (1993); Swerts, Collier e Terken (1994), Swerts (1997), Donzel (1999), Oliveira Jr (2000), Arim, Costa e Freitas (2003), dentre outros.

Tal abordagem intuitiva evitou o problema da circularidade apontado por Ladd (1986) e garantiu que a percepção do investigador na segmentação dos agrupamentos numéricos não fosse influenciada por aspectos acústico-visuais. Além disso, estudos prévios (SWERTS, 1997; MO, COLE; LEE, 2008; SILVA; OLIVEIRA JR, 2011; OLIVEIRA JR; CRUZ; SILVA, 2012) comprovaram que: i) a percepção de ouvintes, ainda que não treinados na identificação de fronteira prosódica, apresenta relação com as pistas fonéticas previstas para essa posição; e ii) quando realizados testes de concordância entre examinadores, treinados ou não, eles concordam de maneira significativa acerca da segmentação de um mesmo enunciado.

Então, no intuito de examinar em que medida a estrutura dos agrupamentos numéricos de CPF é reconhecida por examinadores (especialistas e leigos) aplicamos o teste estatístico Kappa Fleiss, seguindo metodologia semelhante a Mo, Cole e Lee (2008) e Oliveira Jr, Cruz e Silva (2012). Os resultados do teste Kappa de concordância entre examinadores apontaram para uma concordância significativa quanto à segmentação dos agrupamentos numéricos, tanto entre examinadores treinados quanto entre não treinados, já que o coeficiente Kappa não apresentou valores abaixo de 0,85 . Sendo assim, procedemos à segmentação e anotação dos dados. A anotação dos dados foi multinível e organizada em camadas no Praat e todos os testes estatísticos foram realizados por meio do software estatístico $R$ (R Core Team, 2016).

Diante dos dados devidamente anotados, a primeira etapa das análises dos agrupamentos consistiu em verificar a distribuição de frequência numérica mais recorrente dos enunciados numéricos de CPF. Nesse caso, por se tratar de variáveis categóricas (número de ocorrências), realizamos o teste estatístico do Qui-quadrado $\left(\chi^{2}\right)$ de Pearson a fim de mostrar se a distribuições numéricas mais recorrentes apresentaram diferença estatisticamente significativa entre elas. Dessa forma, a frequência numérica mais significativa representaria o padrão de enunciação do agrupamento numérico de CPF e, sobre tais dados de fala realizamos a análise acústica.

Todos os valores correspondentes ao parâmetro da f0 foram extraídos a partir do script AnalyseTier (HIRST, 2012), utilizado também em estudos prévios (HOFFMANN, 2011; ARGUEDAS; MARCO, 2014; CABEDO, 2014, dentre outros). E, por se tratar de variáveis quantitativas, utilizamos o teste estatístico de análise da variância (ANOVA) de Fisher para verificar se houve diferença significativa entre os níveis de análise. Entretanto, quando analisamos variáveis com mais de dois níveis utilizamos, após o teste ANOVA, o teste estatístico de comparação múltipla de Tukey a fim de comparar os níveis dois a dois e identificar entre quais níveis de análise reside a diferença constatada no teste ANOVA.

Para obter os valores referentes à análise acústica da frequência fundamental (variação da f0, declínio da f0 e diferença de tom) das unidades prosódicas a partir do AnalyseTier, utilizamos a tira (camada) gerada na etapa da segmentação e anotação dos dados para identificar as unidades prosódicas.

A variação da f0, medida aqui como pitch range, foi utilizada nessa pesquisa como parâmetro para análise da frequência fundamental, uma vez que, de acordo com Pike (1945), 
Crystal (1969), Bolinger (1972), Pierrehumbert (1980) e Ladd (2008[1996]) a variação da f0 é um dos melhores parâmetros para análise da frequência fundamental, cujos valores foram expressos em semitons (relativos a $100 \mathrm{~Hz}$ ), seguindo estudos anteriores (SWERTS, 1997; OLIVEIRA JR, 2000; HOLM, 2003; CUMBERS, 2013, por exemplo).

Gussenhoven et al (1997), Ouden e Terken (2001) e Ouden (2004) afirmam que a literatura oferece pelo menos duas abordagens para medida do pitch range, uma que considera mais apropriada a utilização do pico mais alto do contorno (LIBERMAN; PIERREHUMBERT, 1984) e outra que afirma ser a diferença entre f0 máxima e f0 mínima a medida mais adequada ('t HART; COLLIER; COHEN, 1990; LADD, 2008[1996]).

Diante da falta de consenso na literatura com relação a qual medida seria mais adequada para análises de pitch range, decidimos optar por utilizar como medida de pitch range valores da f0 máxima, na análise de variação da f0 nas unidades prosódicas e no agrupamento numérico como um todo, seguindo abordagem proposta por Liberman e Pierrehumbert (1984), uma vez que ela também foi utilizada posteriormente por outros autores (SWERTS, 1997; OLIVEIRA JR, 2000). Além disso, Ouden (2004), ao comparar ambas as medidas, conclui que o valor do pico mais alto consiste em medida mais apropriada de pitch range de toda a unidade prosódica.

A frequência fundamental também foi analisada no que se refere à declinação por meio da diferença da f0 máxima entre a primeira sílaba tônica (PST) e a última sílaba tônica (UST) de cada unidade prosódica, a fim de verificar se houve um declínio da f0 em cada unidade. Estudos anteriores (SWERTS, 1997; OLIVEIRA JR, 2000, por exemplo) mostram, ao analisar sentenças declarativas neutras não numéricas, que realmente a frequência fundamental tende a declinar no curso de um enunciado, mas geralmente ela é reiniciada em "junções" (fronteiras) no fluxo da informação. Isso implica que entre unidades prosódicas adjacentes há uma diferença de tom que indica variação da f0 maior no início da unidade seguinte do que no final da anterior.

Por essa razão, também analisamos a diferença de tom, que foi medida a partir de valores de pitch range entre duas unidades adjacentes, seguindo Oliveira Jr (2000). Para isso, comparamos valores da f0 máxima entre a última sílaba tônica de uma unidade prosódica e a primeira sílaba tônica da unidade seguinte. Dessa forma, pudemos verificar se ocorreu em nossos dados o que Swerts (1997) denominou de descontinuidade melódica, ou seja, quando ocorre um reinício da frequência fundamental (pitch reset), com a subida da f0, a medida que uma nova unidade prosódica é enunciada. Vale destacar que utilizamos os termos pitch range e pitch reset para indicar respectivamente gama da $\mathrm{f0}$ e reinício da f0, mesmo sabendo que o termo "pitch" corresponde ao correlato perceptual da f0, porque as pesquisas em que nos embasamos para a análise utilizam tais termos.

\section{Resultados e discussão}

Nessa seção, apresentamos análises da distribuição de frequência numérica, bem como procedemos à análise acústica da f0 (variação de f0, declínio de f0 e diferença de tom) das unidades prosódicas dos números de CPF, enunciados de forma espontânea.

Para a enunciação espontânea dos números de CPF, solicitamos apenas que os participantes fornecessem seus números pessoais, sem consulta, durante uma pequena entrevista em que outros agrupamentos numéricos também foram solicitados, como número de CEP e de telefone. Dos 121 participantes que compuseram o corpus dessa pesquisa, 10 não forneceram seus números pessoais de CPF e, por essa razão, obtivemos 111 arquivos de áudio gerados a partir da enunciação espontânea dos números de CPF dos participantes. 


\subsection{Distribuição de frequências do CPF}

A partir desses dados, realizamos o levantamento das estratégias de enunciação espontânea utilizadas pelos participantes, no que se refere à distribuição numérica, durante a enunciação dos números de CPF. Desse modo, obtivemos 90,1\% dos agrupamentos enunciados em três unidades ternárias finalizando com uma binária. Percebemos, portanto, que embora o conjunto de números que constituem o CPF de cada informante seja sempre diferenciado, parece haver uma tendência voltada a uma distribuição numérica que os organiza em 3-3-3-2, como é possível observar na Tabela 1.

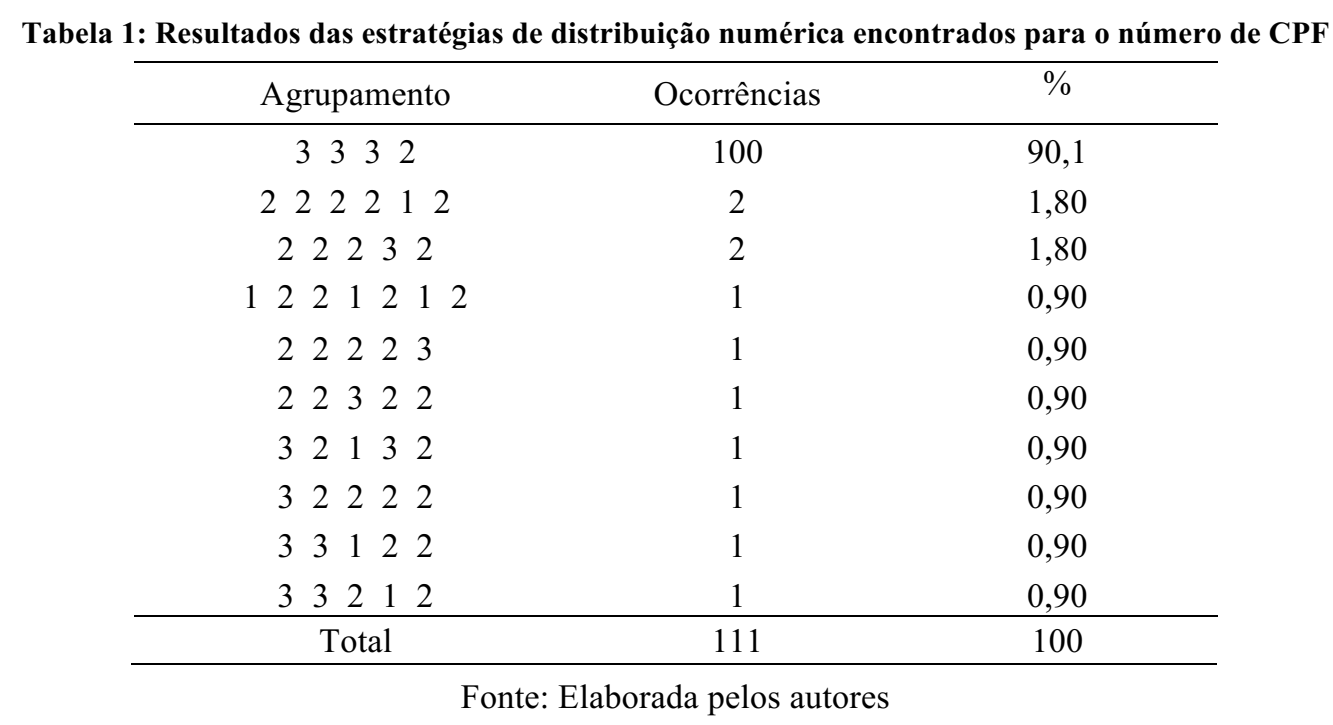

Além disso, esse resultado reforça a ideia de que a disposição gráfica como o agrupamento numérico aparece no documento pode interferir na forma como o mesmo é enunciado. As três distribuições numéricas mais recorrentes na enunciação de dados pessoais de CPF são estatisticamente diferentes $\left(\chi^{2}=184,69, p<0,05, d f=2\right)$, ou seja, a distribuição numérica mais frequente, 3-3-3-2, representa o padrão de enunciação de números de CPF em nosso corpus.

As seções seguintes apresentam resultados das análises acústicas das unidades prosódicas resultantes da enunciação espontânea do CPF, no que se refere à variação da f0, declínio da f0 e diferença de tom. Conforme salientamos na metodologia, procedemos a essas análises de características prosódicas com dados provenientes da distribuição numérica mais frequente, 3-3-3-2. Por essa razão, nas seções seguintes, foram analisados 100 agrupamentos numéricos de CPF, enunciados de forma espontânea. Tais valores foram extraídos por meio da utilização do script AnalyseTier (HIRST, 2012).

\subsection{Variação da f0 nas unidades prosódicas do CPF}

Nessa seção, apresentamos resultados a respeito da variação da f0 nas unidades prosódicas da enunciação espontânea dos números de CPF. Assim, conforme explicitamos na metodologia, a variação da f0 é medida aqui como pitch range, tomando como correlato acústico o valor da f0 máxima (SWERTS, 1997; OLIVEIRA JR, 2000; OUDEN, 2004). O Gráfico 1 mostra a relação entre os valores de f0 nas unidades prosódicas da enunciação espontânea de CPF. 
Gráfico 1: Boxplot da relação entre variação de f0 e unidades prosódicas (01, 02, 03, 04) do CPF



Fonte: Elaborado pelos autores

O teste estatístico ANOVA mostrou que a distribuição dos valores da f0 entre as unidades prosódicas que estão sendo comparadas apresenta uma diferença estatisticamente significativa, $F(3,396)=3,127, p<0,05$. O teste estatístico de comparações múltiplas de Tukey foi realizado e obtivemos os seguintes valores de $p$ demonstrados na Tabela 2.

Tabela 2: Valores de p, relativos à variação de fo, resultantes do teste Tuk
comparando as unidades prosódicas do CPF
\begin{tabular}{cc} 
Unidades prosódicas & Valor $p$ \\
\hline CPF_P_02-CPF_P_01 & 0,6311471 \\
CPF_P_03-CPF_P_01 & 0,4599477 \\
CPF_P_04-CPF_P_01 & 0,0134560 \\
CPF_P_03-CPF_P_02 & 0,9930423 \\
CPF_P_04-CPF_P_02 & 0,2538635 \\
CPF_P_04-CPF_P_03 & 0,3945946 \\
\hline
\end{tabular}

Fonte: Elaborada pelos autores

Os resultados do teste Tukey revelaram haver diferença estatisticamente significativa de valores da f0, apenas entre a primeira e a quarta unidades prosódicas. Portanto, parece não haver diferenças relevantes de variação da f0 entre as unidades prosódicas na enunciação de números de CPF.

De acordo com Kent e Read (2015), há um declínio da frequência fundamental no interior das unidades prosódicas, mas que essa variação da f0 dentro das unidades são quase constantes quando comparamos uma unidade com a outra. Afirmam ainda que tal declinação torna-se maior com o comprimento do enunciado decrescente. Em seguida, analisamos o declínio da f0 nos números de CPF.

\subsection{Declínio da f0 nas unidades prosódicas do CPF}

O declínio da f0 abordada nessa seção foi observada por meio da diferença da f0 máxima entre a primeira sílaba tônica (PST) e a última sílaba tônica (UST) de cada unidade prosódica, a fim de verificar se houve um declínio da f0 em cada unidade. A esse respeito, a literatura prévia, que aborda sentenças declarativas neutras não numéricas, afirma que os valores da frequência fundamental máxima no final de uma unidade prosódica tendem a ser mais baixos do que no início da unidade (SWERTS e GELUYKENS, 1994; SWERTS, 1997; 
OLIVEIRA JR, 2000; LUCENTE, 2012; BARBOSA; MADUREIRA, 2015; KENT e READ, 2015; SERRA, 2009).

Isso acontece porque, de acordo com alguns autores (SORENSEN e COOPER, 1980; STERNBERG et al, 1980; 't HART, COLLIER e COHEN, 1990; OUDEN, 2004), os falantes controlam voluntariamente essa declinação, regulando-a de modo a iniciar a sentença com valores altos de f0 no intuito de manter perceptivamente uma declinação saliente. Além disso, Ferreira Netto (2007) apresentou resultados para uma análise da declinação no português do Brasil que converge com esses estudos anteriores, evidenciando que a fala precisa ser préprogramada para ajustar sua duração e sua variação de frequências. Sugere que o falante precisa prever a duração de sua sentença, iniciando com um tom que seja alto o suficiente para que possa ser concluído com um tom baixo.

Abordamos nessa seção a análise do declínio da frequência fundamental nas unidades prosódicas dos números de CPF enunciados espontaneamente. Percebemos um declínio da f0, no interior de cada unidade prosódica e ao considerar todo o agrupamento numérico do CPF, conforme evidenciado no Gráfico 2.

Gráfico 2: Boxplot do declínio da f0 no interior das unidades prosódicas do CPF



Fonte: Elaborado pelos autores

Esse declínio da f0 evidencia que os valores da f0 máxima nas últimas sílabas tônicas são menores do que os das primeiras sílabas tônicas no interior de todas as unidades prosódicas e também se compararmos o agrupamento numérico como um todo. Ao realizar o teste ANOVA verificamos que esse declínio da f0, evidenciado no gráfico, é significativo em todas as unidades prosódicas, conforme resultados estatísticos apresentados na Tabela 3.

Tabela 3: Resultados estatísticos relativos ao declínio de f0 no interior das unidades prosódicas do CPF

\begin{tabular}{cc}
\hline Unidades prosódicas & Teste ANOVA \\
\hline 01 & $F(1,198)=5,13, p<0,05$ \\
02 & $F(1,198)=4,14, p<0,05$ \\
03 & $F(1,198)=6,62, p<0,05$ \\
04 & $F(1,198)=6,35, p<0,05$ \\
\hline
\end{tabular}

Fonte: Elaborada pelos autores 
Considerando todo o agrupamento numérico, verificamos que o declínio da f0 é ainda mais significativo, $F(1,198)=19,87, p<0,05$. Isso corrobora a afirmação de Kent e Read (2015) de que a maior queda de f0 marca o fim de um enunciado e quedas menores antecedendo-o indicam que o falante pretende continuar o enunciado, marcando unidades prosódicas.

Tais resultados apontam para um comportamento de f0 típico de sentenças declarativas neutras no português brasileiro que apresenta um início marcado pela subida de f0 e um final de sentença marcado pela queda de f0 (MORAES, 1998; LUCENTE, 2012; BARBOSA; MADUREIRA, 2015; SOUZA, 2007; SERRA, 2009).

Estudos anteriores (PIJPER e SANDERMAN, 1994; SWERTS, 1997; OLIVEIRA JR, 2000, por exemplo) mostram que realmente a frequência fundamental tende a declinar no curso de um enunciado não numérico, mas geralmente ela é reiniciada em "junções" (fronteiras) no fluxo da informação. Isso implica que entre unidades prosódicas adjacentes há uma diferença de tom que indica variação de f0 maior no início da unidade seguinte do que no final da anterior, conforme também podemos observar no Gráfico 2. Resultados a esse respeito são apresentados na seção seguinte.

\subsection{Diferença de tom entre as unidades prosódicas adjacentes do CPF}

A diferença de tom foi medida a partir de valores de pitch range entre duas unidades adjacentes, seguindo Oliveira $\operatorname{Jr}$ (2000). Para isso, comparamos valores da f0 máxima entre a última sílaba tônica de uma unidade e a primeira sílaba tônica da unidade seguinte, nos enunciados de CPF.

Essa seção apresenta resultados sobre o reinício da frequência fundamental na enunciação espontânea dos números de CPF. O Gráfico 3 sugere que realmente houve uma diferença de tom entre as unidades adjacentes evidenciando uma descontinuidade melódica (SWERTS, 1997), ou seja, um pitch reset, à medida que uma nova unidade prosódica é enunciada.

Gráfico 3: Boxplot da diferença de tom entre unidades prosódicas adjacentes do CPF

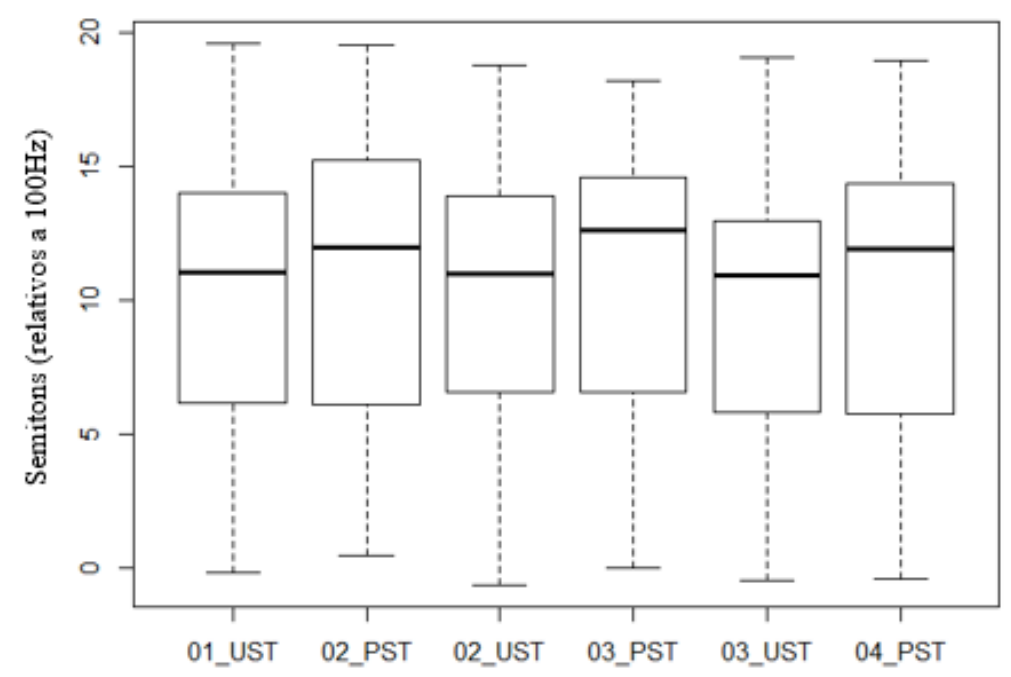

Fonte: Elaborado pelos autores

No Gráfico 3 fica evidente o reinício da f0 (pitch reset), com a subida da f0, a cada nova unidade prosódica. Fato já constatado no português brasileiro para sentenças não numéricas (OLIVEIRA JR, 2000; SERRA, 2009; LUCENTE, 2012), e também em outras 
línguas, como por exemplo, o holandês (SWERTS, 1997; PIJPER; SANDERMAN, 1994; SWERTS; GELUYKENS, 1994), e o sueco (SWERTS; STRANGERT; HELDNER, 1996).

A Tabela 4 mostra que não houve diferença significativa de variação da f0 entre as unidades adjacentes de enunciação espontânea do CPF, de acordo com os resultados do teste estatístico ANOVA.

Tabela 4: Resultados estatísticos relativos à diferença de tom entre unidades adjacentes do CPF

\begin{tabular}{cc}
\hline Diferença de tom & Teste ANOVA \\
\hline 01_UST -02_PST & $F(1,198)=2,50, p>0,05$ \\
02_UST -03_PST & $F(1,198)=3,46, p>0,05$ \\
03_UST -04 _PST & $F(1,198)=1,83, p>0,05$ \\
\hline
\end{tabular}

Fonte: Elaborada pelos autores

Portanto, embora seja visível o reinício da frequência fundamental entre as unidades prosódicas adjacentes, no Gráfico 3, essa diferença não se mostrou estatisticamente significativa na enunciação espontânea dos números de CPF. Tal constatação talvez seja explicada pelo fato de estarmos analisando enunciados curtos, já que, conforme Kent e Read (2015), sentenças mais longas apresentam maiores valores de pitch reset enquanto que em sentenças mais curtas as declinações de cada unidade prosódica permanecem bastante constantes, como constatamos na análise de variação da f0.

\section{Conclusão}

Observamos que a distribuição numérica padrão dos agrupamentos de CPF foi representada pela forma numérica 3-3-3-2, mantendo uma relação direta com a forma gráfica, repetindo a estrutura constante no documento (NNN.NNN.NNN-NN). Esse comportamento corrobora com estudos anteriores (SPERLING, 1988; HALFORD; COWAN; ANDREWS, 2007; COWAN, 2010) que ao abordarem sobre dígitos, apontam como estratégia de armazenamento, uma preferência de organização binária, subdividindo o agrupamento em dois ou até três elementos.

Além disso, os resultados dos números de CPF descritos neste trabalho confirmaram estudos anteriores, como o de Baumann e Trouvain (2001), por exemplo, que propõe que, independentemente da língua, o agrupamento de números de telefone é peculiar em vários aspectos, pois geralmente não são tratados como uma única unidade, como unidades monetárias ou problemas aritméticos. Os autores identificaram que as unidades preferidas são binárias ou ternárias, inserindo às vezes, dígito único. Mussiliyu (2014) também encontrou resultados similares em sua análise de números de telefone com oito dígitos.

Nas análises acústicas referentes à característica prosódica de variação da f0 nas unidades prosódicas constatamos que, de modo geral, não houve diferença estatisticamente significativa entre as unidades. Provavelmente porque essa variação da f0 dentro das unidades prosódicas é quase constante se compararmos uma unidade prosódica com outra (KENT; READ, 2017).

Swerts (1997), Oliveira Jr. (2000), Kent e Read (2007) afirmam que é comum haver uma queda da frequência fundamental dentro de cada unidade prosódica, fato que também constatamos em nossos dados. Verificamos que, embora não tenhamos encontrado diferença significativa no declínio da f0, observamos o declínio da f0 dentro das unidades prosódicas através do comportamento da f0 máxima no início e no final dessas unidades. Ao considerarmos o agrupamento como um todo, percebemos que esse declínio foi ainda mais significativo. Nossos achados confirmaram que a frequência fundamental tende a declinar no 
curso de um enunciado numérico, assim como ocorre em enunciados não numéricos, mas geralmente ela é reiniciada nas fronteiras. Isso implica que há uma diferença de tom entre unidades prosódicas adjacentes.

Procedemos à mensuração da diferença de tom a partir de valores de pitch range entre duas unidades adjacentes, seguindo Oliveira Jr (2000), no intuito de observar a presença de reinício da f0 (pitch reset) a cada unidade prosódica enunciada. Verificamos que, apesar de não ter apresentado diferença significativa, realmente houve uma diferença de tom entre as unidades adjacentes em todos os agrupamentos numéricos analisados. A não observação de efeitos estatisticamente significativos talvez seja explicada pelo fato de estarmos analisando enunciados curtos, já que, conforme Kent e Read (2007), sentenças mais longas apresentam maiores valores de pitch reset enquanto que em sentenças mais curtas as declinações no interior de cada unidade prosódica permanecem bastante constantes. Portanto, os resultados mostram uma tendência para aquilo que Swerts (1997) denominou de descontinuidade melódica, quando ocorre um reinício da frequência fundamental, com a subida da f0, a medida que uma nova unidade prosódica é enunciada.

Os resultados da presente investigação experimental demonstraram que os falantes do português brasileiro são conscientes da existência de uma estrutura numérica subjacente, e que essa consciência é evidenciada através do uso sistemático de vários elementos prosódicos. Ou seja, os números de CPF agrupados em estruturas fixas apresentam uma estrutura razoavelmente previsível, que é sistematicamente sinalizada por diversas pistas prosódicas, sobretudo as de natureza entoacional.

Nossos achados confirmam a hipótese de que os enunciados numéricos apresentam características prosódicas semelhantes às apontadas em estudos prévios para sentenças declarativas neutras não numéricas no português brasileiro. Além disso, os resultados do teste Kappa de concordância ratificam outra hipótese, qual seja, a de que o falante possui intuitivamente a capacidade de perceber o local das fronteiras prosódicas, assim como já foi comprovado com sentenças não numéricas em investigações anteriores (SWERTS, 1997; MO; COLE; LEE, 2008; OLIVEIRA JR; CRUZ; SILVA, 2012).

Apesar dessa pesquisa objetivar reduzir uma lacuna na literatura reservada à descrição prosódica de números nominais no português brasileiro, entendemos que ainda há muito a ser feito, sobretudo no que diz respeito à aplicabilidade dos dados que estão disponibilizados para futuras investigações.

\section{Referências}

ALMEIDA, Ayane N. S. de. Análise prosódica de agrupamentos numéricos no português do Brasil. Tese de Doutorado, Universidade Federal de Alagoas, Maceió, 2017.

AMINO, Kanae; OSANAI, Takashi. Realisation of the prosodic structure of spoken telephone numbers by native and non-native speakers of Japanese. Proceedings of the 17th International Congress of Phonetic Sciences. Honk kong, China, ICPhS, p. 236-239, 2011. ARGUEDAS, Maria Estellés; MARCO, Marta Albelda. Evidentials, politeness and prosody in Spanish: A corpus analysis. Journal of Politeness Research, vol. 10, n. 1, p. 29-62, 2014.

ARIM, Eva; COSTA, Fancisco; FREITAS, Tiago. A study on the reliability of two discourse segmentation models. Proceedings of the 6th international conference on Computational processing of the Portuguese language, Faro, Portugal, p. 70-77, 2003.

BARBOSA, Plínio A.; MADUREIRA, Sandra. Manual de fonética acústica experimental: aplicação a dados do português. São Paulo: Cortez, 2015.

BARNWELL, Brendan. Perception of prosodic boundaries by untrained listeners. In: REED, Beatrice Szczepek; RAYMOND, Geoffrey (eds.). Units of Talk - Units of Action. Amsterdam: John Benjamins, 2013, p. 125-165. 
BARTH-WEINGARTEN, Dagmar. From "intonation units" to cesuring - an alternative approach to the prosodic-phonetic structuring of talk-in-interaction. In: REED, Beatrice Szczepek; RAYMOND, Geoffrey (eds.). Units of Talk - Units of Action. Amsterdam: John Benjamins, 2013, p. 91-124.

BAUMANN, Stefan; TROUVAIN, Jürgen. On the prosody of German telephone numbers. Proceedings of the 2th INTERSPEECH and 7th European Conference on Speech Communication and Technology (EUROSPEECH 2001), Aalborg, Denmark, p. 557-560, 2001.

BLAAUW, Eleonora. On the perceptual classification of spontaneous and read speech. Utrecht University, Utrecht, 1995.

BOERSMA, Paul. Praat, a system for doing phonetics by computer. Glot International, vol. 5, n. 9-10, p. 341-345, 2001.

BOLINGER, Dwight L. Accent is predictable (if you're a mindreader). Language, vol. 48, n. 3, p. 633-644, 1972.

. Intonation and its Uses: Melody in Grammar and Discourse. Stanford: Stanford University Press, 1989.

CABEDO, Adrián. Relación entre el grado de certeza y los valores de F0 y duración silábica en algunos adverbios terminados en -mente. Revista Signos, Estudios de Linguística, vol. 47, n. 85, p. 196-216, 2014.

CHAFE, Wallace. Discourse, Consciousness, and Time: The Flow and Displacement of Conscious Experience in Speaking and Writing. Chicago: University of Chicago Press, 1994.

COLLIER, René. On the communicative function of prosody: some experiments. IPO Annual Progress Report, vol. 28, p. 67-75, 1993.

COWAN, Nelson. The Magical Mystery Four: How is Working Memory Capacity Limited, and Why?. Current Directions in Psychological Science, vol. 19, n. 1, p. 51-57, 2010.

CRUTTENDEN, Alan. Intonation. 2.ed. Cambridge: Cambridge University Press, 1997[1986].

CRYSTAL, David. Prosodic systems and intonation in English. Cambridge: Cambridge University Press, 1969.

CUMBERS, Bree A. Perceptual correlates of acoustic measures of vocal variability. Thesis (Master of Science). University of Wisconsin-Milwaukee, 2013.

DONZEL, Monique Elisabeth van. Prosodic Aspects of Information Structure in Discourse. Amsterdam: Holland Academic Graphics, 1999.

FERREIRA NETTO, Waldemar. Declinação frasal na língua portuguesa. 55 $^{\circ}$ Seminário do Grupo de Estudos Linguísticos (GEL). Universidade de Franca, 2007.

GUSSENHOVEN, Carlos et al. The perceptual prominence of fundamental frequency peaks. Journal of the Acoustical Society of America, vol. 102, n. 5, p. 3009-3022, 1997.

HALFORD, Graeme S.; COWAN, Nelson; ANDREWS, Glenda. Separating Cognitive Capacity from Knowledge: A New Hypothesis. Trends in Cognitive Sciences, vol. 11, n. 6, p. 236-242, 2007.

HIRST, Daniel. Analyse tier PRAAT script, 2012. Disponível em: <https://uk.groups. yahoo.com/neo/groups/praat-users/files/Daniel_Hirst/analyse_tier.praat $>$. Acesso em: $15 / 05 / 2013$.

HOFFMANN, Thomas. The Black Kenyan English vowel system: An acoustic phonetic analysis. English World-Wide, vol. 32, n. 2, p. 147-173, 2011.

HOLM, Snefrid. Individual use of acoustic parameters in read and spontaneous speech. Phonum, vol. 9, p. 157-160, 2003.

KENT, Ray D.; READ, Charles. Análise acústica da fala. Tradução Alexsandro Rodrigues Meireles. São Paulo: Cortez, 2015. 
LADD, D. Robert. Intonational phrasing: the case for recursive prosodic structure. Phonology, vol. 3, n.1, p. 311-340, 1986. 2008[1996].

Intonational Phonology. 2.ed. Cambridge: Cambridge University Press, LIBERMAN, Mark; PIERREHUMBERT, Janet B. Intonational Invariance under Changes in Pitch Range and Length. In: ARONOFF, Mark; OEHRLE, Richard T. (eds.). Language Sound Structure. Cambridge: MIT Press, 1984, p. 157-233.

LIEBERMAN, Philip. Intonation, Perception, and Language. Cambridge: MIT Press, 1967.

LUCENTE, Luciana. Aspectos dinâmicos da fala e da entoação no português brasileiro. Tese de doutorado apresentada ao Programa de Pós-graduação em Linguística do Instituto de Estudos da Linguagem da Universidade Estadual de Campinas. Campinas, SP, 2012.

MO, Yoonsook; COLE, Jennifer; LEE, Eun-Kyung. Naïve listeners' prominence and boundary perception. Proceedings of the 4th International Conference on Speech Prosody, Campinas, Brazil, p. 735-738, 2008.

MOKBEL, Chafic; MAUUARY, Laurent; KARRAY, Lamia; JOUVET, Denis; MONNÉ, Jean; SIMONIN, Jacques; BARTKOVA, Katarina. Towards improving ASR robustness for PSN and GSM telephone applications, Speech Communication, 23, 1-2, p. 141-159, 1997.

MORAES, João Antônio de. Intonation in Brazilian Portuguese. In: HIRST, Daniel; DI CRISTO, Albert. Intonation Systems: a survey of twenty languages. Cambridge: Cambridge University Press, 1998, p. 179-194.

MUSILIYU, Oyedeji. Características prosódicas dos números telefônicos no português brasileiro. Dissertação (Mestrado) - Programa de Pós-graduação em Letras e Linguística. Maceió: Universidade Federal de Alagoas, 2014.

OLASZI, Péter. Analysis of Written and Spoken Form of Hungarian Numbers for TTS Applications, International Journal of Speech Technology, 3, 3, p. 177-186, 2000.

OLIVEIRA JR., Miguel. Prosodic features in spontaneous narratives. Thesis (Doctor of Philosophy). Department of Linguistics, Simon Fraser University, Vancouver, 2000.

Aspectos técnicos na coleta de dados linguísticos orais. In: FREITAG, Raquel

Meister Ko. Metodologia de Coleta e Manipulação de dados em Sociolinguística. São Paulo: Blucher, 2014.

. A Study on Speech Rate as a Prosodic Feature in Spontaneous Narrative. Alfa, vol.

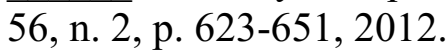

OLIVEIRA JR., Miguel; CRUZ, Regina; SILVA, Ebson Wilkerson. A relação entre a prosódia e a estrutura de narrativas espontâneas: um estudo perceptual. Revista Diadorim, vol. 12, p. 38-53, 2012.

OUDEN, Hanny den. Prosodic realizations of text structure. Thesis (Doctor of Philosophy) - Universiteit van Tilburg, Netherlands. Enschede: Ipskamp, 2004.

OUDEN, Hanny den; TERKEN, Jacques. Measuring Pitch Range. Proceedings of the 2th INTERSPEECH and 7th European Conference on Speech Communication and Technology (EUROSPEECH 2001), Aalborg, Denmark, p. 91-94, 2001.

PIJPER, Jan Roelof de; SANDERMAN, Angelien. On the perceptual strenght of prosodic boundaries and its relation to supresagmental cues. Journal of the Acoustical Society of America, vol. 96, n. 4, p. 2037-2047, 1994.

PIKE, Kenneth Lee. The Intonation of American English. Ann Arbor: University of Michigan Press, 1945.

R Core Team. R: A language and environment for statistical computing. R Foundation for Statistical Computing, Vienna, Austria, 2016. Disponível em: < https://www.r-project.org/>. Acesso em: 21/06/2016. 
REED, Beatrice Szczepek. Intonation phrases in natural conversation: a participants' category? In: BARTH-WEINGARTEN, Dagmar; REBER, Elisabeth; SELTING, Margaret (eds.). Prosody in Interaction. Amsterdam: John Benjamins, 2010, p. 191-212.

Analysing Conversation: an introduction to prosody. Houndmills: Palgrave Macmillan, 2011.

RODRIGUES, Frederico. Reconhecimento Robusto de Dígitos e Números Naturais. Tese de doutorado. Universidade Técnica de Lisboa, Portugal, 2001.

SERRA, Carolina Ribeiro. Realização e percepção de fronteiras prosódicas no português do Brasil: fala espontânea e leitura. Tese de doutorado apresentada ao Programa de Pósgraduação em Letras Vernáculas da Universidade Federal do Rio de Janeiro. Rio de Janeiro, 2009.

SILVA, Ebson Wilkerson; OLIVEIRA JR, Miguel. A percepção dos elementos prosódicos como marca de estruturação de narrativas espontâneas: um estudo preliminar. Anais do III Colóquio Brasileiro de Prosódia da Fala. Belo Horizonte, 2011, p. 15-17.

SORENSEN, John M.; COOPER, William E. Syntactic coding of fundamental frequency in speech production. In: COLE, Ronald Allan. (ed.). Perception and production of fluent speech. New Jersey: Lawrence Erlbaum Associates, 1980, p. 399-440.

SOUZA, Karine Kellvia de. Análise do fenômeno da declinação na entonação de sentenças declarativas isoladas dos falantes do português brasileiro. Dissertação (Mestrado) - Programa de Pós-graduação em Estudos Linguísticos da Faculdade de Letras. Belo Horizonte: Universidade Federal de Minas Gerais, 2007.

SPERLING, George. The magical number seven: Information processing then and now. In: HIRST, William. The making of cognitive Science: Essays in honor of George A. Miller. Cambridge, UK: Cambridge University Press, 1988, p. 71-80.

STERNBERG, Saul et al. Motor programs in rapid speech: additional evidence. In: COLE, Ronald Allan. (ed.). Perception and production of fluent speech. New Jersey: Lawrence Erlbaum Associates, 1980, p. 507-534.

SWERTS, Marc. Prosodic features at discourse boundaries of different strength. Journal of the Acoustical Society of America, vol. 101, n. 1, p. 514-521, 1997.

SWERTS, Marc; COLLIER, René. On the controlled elicitation of spontaneous speech. Speech Communication, vol. 11, n. 4-5, p. 463-468, 1992.

SWERTS, Marc; COLLIER, René; TERKEN, Jacques. Prosodic predictors of discourse finality in spontaneous monologues. Speech Communication, vol. 15, n. 1-2, p. 79-90, 1994. SWERTS, Marc; GELUYKENS, Ronald. Prosody as a Marker of Information Flow in Spoken Discourse. Language and Speech, vol. 37, n. 1, p. 21-43, 1994.

SWERTS, Marc; STRANGERT, Eva; HELDNER, Mattias. $F_{0}$ declination in read-aloud and spontaneous speech, Proceedings of the 4th International Conference on Spoken Language (ICSLP 96), Philadelphia, p. 1501-1504, 1996.

't HART, Johan; COLLIER, René; COHEN, Antonie. A perceptual study of intonation: an experimental phonetic approach to speech melody. Cambridge: Cambridge University Press, 1990.

WANG, Chao; SENEFF, Stephanie. A study of tones and tempo in continuous Mandarin digit strings and their application in telephone quality speech recognition. Proceedings of the International Conference on Spoken Language Processing (ICSLP 98), Sydney, Australia, p. 695-698, 1998.

WIESE, Heike. Numbers, Language, and the human mind. Cambridge: Cambridge University Press, 2003. 